\title{
Efectividad de la campaña de comunicación del Ministerio de Salud para la prevención de la leptospirosis. Municipio de Achuapa (León)
}

\author{
Dra. Maryury Stella Pérez Vargas
}

Recepcionado: 20 abril, 2013 / Aceptado: 15 de julio, 2013

\section{RESUMEN}

Se realizó un estudio mixto (corte transversal combinado con metodología cualitativa) en el municipio de Achuapa, con el objetivo de determinar la efectividad de la Campaña de Comunicación del Ministerio de Salud para la prevención de la Leptospirosis en el Municipio, durante el tercer cuatrimestre del 2010. La campaña de publicidad se genera como parte de las acciones contempladas por el Gobierno de Nicaragua, ante la necesidad de declarar en alerta sanitaria al país por la difícil situación presentada en el mes de septiembre del 2010. El método de trabajo consistió en la aplicación de una encuesta domiciliar a una muestra estratificada de 369 habitantes y distribuida de manera proporcional en las cinco comarcas que conforman el Municipio de Achuapa, en la realización de una entrevista en profundidad a la epidemióloga municipal y en la realización de dos grupos focales con la población del Municipio de Achuapa. Se encontró una comprensión muy alta de la población sobre la Leptospirosis y sus formas de transmisión. El reconocimiento y prácticas de la comunidad sobre las medidas de mitigación, control y prevención sobre la leptospirosis, recomendadas por el Ministerio de Salud presentaron una comprensión muy alta en la población. La percepción que tiene la población de Achuapa referente al trabajo desempeñado por el Ministerio de Salud es valorada como buena. El papel de los medios de comunicación fue valorado como bueno, siendo la radio el principal medio utilizado para las campañas de salud. Se recomienda al MINSA en conjunto con los actores locales realizar un seguimiento a mediano plazo de la campaña de comunicación, con el objetivo de mejorar la calidad de la intervención y desarrollar medidas para la mejora continua de las estrategias de educación, comunicación e información.

Palabras claves: leptospirosis, campaña de educación, mitigación, control, comunicación.

\section{INTRODUCCIÓN}

En Nicaragua la leptospirosis ha estado adquiriendo una gran relevancia en los últimos años, específicamente a partir de 1995, año de presentación del brote conocido como "fiebre de Achuapa".

Al 24 de septiembre del 2010 se habían registrado en el país 194 personas contagiadas, en este mismo año se registraron 16 muertes por leptospirosis en la región del occidente del país (INFOMED, 2010).

Ante la situación presentada las autoridades declararon la alerta sanitaria el 29 de septiembre del 2010, en todo el territorio nacional, instruyendo a todas las Autoridades de Salud del país a implementar el Plan Nacional para la Prevención y Respuesta que contemplaba dentro de sus acciones ejecutar un amplio programa de comunicación social. (SINAPRED, 2010) Organismos como el Ministerio de Salud Pública, el Ministerio de Agricultura y Forestal, El Ministerio de Educación, las Universidades y las Alcaldías, concentraron sus esfuerzos en ejecutar el programa de comunicación social, dirigida a educar a la población a través de medios de comunicación locales y nacionales sobre síntomas de la enfermedad y de gravedad, sobre medidas preventivas de la enfermedad y promoción de la salud a través de charlas, volantes, viñetas radiales y televisivas, afiches e informar a la comunidad escolar, estudiantes, maestros y padres de familia de las medidas preventivas de la enfermedad y promoción de la salud. 
Con esta investigación se determinará la efectividad de la campaña de comunicación del Ministerio de Salud para la prevención de la leptospirosis en el Municipio de Achuapa, durante el tercer cuatrimestre del año 2010, determinando el grado de conocimiento de la población respecto a la enfermedad y la incidencia de la campaña en la reducción de casos a partir de su implementación. En investigación epidemiológica, se define la efectividad como la capacidad de una intervención de producir el efecto deseado en condiciones reales o habituales. (Dictionary of epidemiology, 1988).

Considerando la alarmante situación que se presentó en la región de occidente de Nicaragua por la presencia de la leptospirosis, se consideró pertinente evaluar si los sistemas y mecanismos de comunicación empleados por las entidades a nivel gubernamental, fueron $\mathrm{y}$ han sido eficaces en cuanto a la trasmisión del conocimiento básico que necesita la población para conocer la enfermedad, detectarla a tiempo, saber qué hacer y en especial evitar que esta se propague.

Para lograr evaluar la afectividad de las campañas de salud emprendidas en el municipio de Achuapa, se realizó el presente estudio de tipo cualicuantitativo, mediante la aplicación de encuestas y el desarrollo de grupos focales, en el cual se estratificó en dos grupos de ubicación (Rural y Urbana), con población de ambos sexos y con personas mayores a 15 años del Municipio de Achuapa.

Con los resultados obtenidos se espera reforzar las estrategias de comunicación que las entidades gubernamentales definan en relación al control, tratamiento y seguimiento de enfermedades transmisibles por contagio de alguna sustancia $u$ otras que afectan la salud de la población, tal como es el Ministerio de Salud, Alcaldía Municipal y las organizaciones comunitarias han implementado para controlar la enfermedad.

\section{MATERIALES Y MÉTODOS}

El presente estudio es de corte transversal con un componente cualitativo con un enfoque cuantitativo con la aplicación de encuestas y enfoque cualitativo basado en la realización de grupos focales y en entrevista en profundidad. El estudio que contempla esta investigación se llevó a cabo en el Municipio de Achuapa ubicado en el departamento de León.

El universo de estudio fue la población urbana y rural del municipio de Achuapa. La población total del municipio de Achuapa es de 14,711.000 habitantes, de los cuales 7,480.000 son mujeres y $7,231.000$ son hombres, para el año 2010. Para este estudio el universo estuvo constituido por la población mayor de 15 años de edad. La población a estudiar fue de 9,600 personas, estratificado de la siguiente manera 8,541 con edad de 15 a 59 años, 272 con edad de 60 a 64 años y 817 con edad de más de 65 años. La población de Achuapa está distribuida en cinco localidades: Achuapa, Barro, Nicolás, Frio y Rosa. A su vez cada localidad cuenta con una unidad de salud.

Para la investigación cuantitativa (entrevistas) se obtuvo una muestra con un $95 \%$ de confianza y un $5 \%$ de error muestral, apartir de una población blanco de 9,600 personas y una frecuencia desconocida de conocimientos y puesta en práctica de la estrategia de comunicación del MINSA, obteniendo un total de 369 encuestas.

Para el estudio cualitativo (grupos focales y entrevista en profundidad), se realizaron dos grupos focales procurando disponer de la representatividad de la población de estudio.

Criterios de selección (Inclusión): Pobladores con edad mayor de 15 años, ser habitante del Municipio de Achuapa, residir en Achuapa por lo menos 1 año. Criterios de restricción (Exclusión): Grupos de 4 a 14 años de edad se excluyen, debido que el enfrentar una entrevista, este grupo de personas se inhiben con facilidad, les da temor contestar y son dependientes en cuanto a su criterio por las personas que los acompañan. Residentes menores de 6 meses, ya que no han sido cubiertos por el período de evaluación del presente estudio. No haber habitado en Achuapa durante el período en que se realizó la investigación. 


\section{RESULTADOS}

Comprensión de la población sobre la leptospirosis y sus formas de transmisión

Al indagar las principales noticias en los temas de salud que la población recuerda durante el III cuatrimestre del 2010, observamos que las noticias sobre leptospirosis fueron las más frecuentes con un $91.3 \%$, seguido delas noticias respecto a las medidas preventivas para la leptospirosis $74.4 \%$ y el resto de la población mencionó el dengue, la influenza.

Al preguntar la razón para decretar la alerta sanitaria el 29 de septiembre del 2010, el 74.8\% de la población identificó a la leptospirosis como la principal razón de la alerta, un $10.6 \%$ indicó no saber o no responder y el resto de la población mencionó dengue, influenza y otras.

Un $85.9 \%$ de los entrevistados reconocen que la leptospirosis es una enfermedad infecciosa, un 9.8\% de los entrevistados indicó no saber o no responder, el resto de los entrevistados mencionó alergia u otro. Un $92.7 \%$ reconoce los principales síntomas de la leptospirosis, el resto de los entrevistados indicó no reconocerlos o no saber. Los síntomas más reconocidos por la población fueron en orden descendente: la fiebre, el dolor de cabeza intenso, ambos con $85.6 \%$, seguido del dolor de cuerpo 72.1\%, malestar general y decaimiento 53.4\%, enrojecimiento de ojos y escalofríos ambos con $49.3 \%$, los entrevistados seleccionaron con menor escala sangrados, tos y color amarillo de la piel.

Un $81.3 \%$ reconoce que la leptospirosis es una enfermedad que puede producir la muerte. Un $18.7 \%$ indica no reconocer que la leptospirosis sea una enfermedad que pueda causar la muerte. Al preguntar dónde acudir si un familiar o amigo se enferma de leptospirosis, un $97.3 \%$ manifestó acudir a un centro de salud y un $2.7 \%$ manifestó acudir a un hospital público.

El $88.1 \%$ de los entrevistados señaló que la forma de transmisión de leptospirosis es a través del consumo de alimentos contaminados con orina de ratas, ratones u otro tipo de animales infectados, el $37.4 \%$ indicó que la forma de transmisión se debía al contacto de las mucosas y la piel lesionada con aguas contaminadas con la orina de animales infectados, el resto de los entrevistados indicó otro o no saber.

Un 95.1\% de la población de estudio identificó a la leptospirosis como una enfermedad muy grave, un $2.7 \%$ no sabía o no respondió al respecto y un $2.2 \%$ indicó calificarla como algo grave. La medida de prevención más identificada fue la de mantener siempre los alimentos tapados para evitar que las ratas y ratones los contaminen con su orina, con un $93.8 \%$, seguida por la medida de no caminar descalzo sobre charcos, lodos y pantanos con un $84 \%$, posteriormente se tiene la medida de limpiar las viviendas y sus alrededores para eliminar criaderos de ratas con un $77.2 \%$, el resto de medidas presentan un porcentaje de reconocimiento mayor al $50 \%$ a excepción de la medida de utilizar botas de hule y pantalón largo en el caso de los trabajadores del campo, con un $46.1 \%$.

\section{Comportamiento y prácticas de la población sobre las medidas de mitigación, control y prevención recomendadas por el Ministerio de Salud}

El $99.7 \%$ manifestó que se deben atender las recomendaciones del MINSA para enfrentar la enfermedad, tan solo el $0.3 \%$ manifestó no atender las recomendaciones del MINSA.

El 97.8\% de la población considera que el gobierno tiene toda la responsabilidad de implementar acciones para prevenir la leptospirosis y el $2.2 \%$ consideró lo contrario. El $100 \%$ de los entrevistados manifestó que se debe atender las recomendaciones del MINSA para la atención y prevención de la enfermedad.

El $100 \%$ de la población expresó que todos debemos contribuir en la prevención de la leptospirosis. La medida de prevención que realiza la población de manera más frecuente es la de limpiar las viviendas y sus alrededores para eliminar criaderos de ratas, identificada en el $95.4 \%$ de los entrevistados. En segundo lugar se identificó 
el mantener siempre sus alimentos tapados para evitar que las ratas y ratones los contaminen con su orina, en un $93.8 \%$ de la población, el resto de las medidas de prevención presenta una realización superior al $50 \%$ a excepción de la medida de evitar nadar en aguas estancadas como pozas, ríos y quebradas.

El 50.1\% de la población señaló que están haciendo caso a algunas de las medidas de prevención que está anunciando el Ministerio de Salud, un 49.3\% indica estar haciendo caso a todas las medidas de prevención y tan solo un $2 \%$ indica estar haciendo caso a pocas de las medidas comunicadas.

Un 51.2\% manifestó que en su casa se realizan algunas de las medidas de prevención recomendadas por el Ministerio de Salud, un 48\% indicó que en su casa se aplican todas las medidas de prevención recomendadas y tan solo un $0.8 \%$ indicó aplicar pocas.

\section{Percepción sobre la gestión de las autoridades de Achuapa referente al trabajo desempeñado por el Ministerio de Salud}

El $99.6 \%$ de los entrevistados considera que el gobierno está informando correctamente acerca del brote de la leptospirosis y solo un $0.4 \%$ considera lo contrario. Al preguntar si ha escuchado u oído sobre la campaña de prevención de la leptospirosis, el $98.9 \%$ respondió afirmativamente, mientras que el $1.1 \%$ respondió negativamente.

El $79.1 \%$ de la población de estudio califica como buena la información que ha dado el Ministerio de Salud sobre la leptospirosis, un $15.5 \%$ la califica como muy buena y un $2.7 \%$ la califica como mala y otra parte igual como mala.

Al indagar la opinión sobre las cifras que han dado a conocer las autoridades sobre el número de personas contagiadas y fallecidas a causa de la leptospirosis, un $52 \%$ consideró que son realistas, mientras el $41,2 \%$ señaló que no se apegan a la realidad. El 95.9\% afirma que el Ministerio de Salud brindó información de forma apropiada y a tiempo sobre la situación de la leptospirosis en el país, el resto de la población indicó que no la brindó de manera adecuada, no sabía o no contestaba.

El $94 \%$ de la población de estudio cree que el Ministerio de Salud garantiza la atención para todas las personas contagiadas con leptospirosis, el resto de la población indica que no sabe o no responde. El $71 \%$ de los entrevistados, evalúa de bueno el desempeño del Ministerio de Salud ante la leptospirosis, un $23.6 \%$ lo evalúa como malo y el resto de la población no sabe o no responde.

Al indagar sobre cómo calificaría la campaña de salud por parte del Ministerio de Salud en cuanto a las medidas de prevención de la leptospirosis en el tercer cuatrimestre del 2010 , un $69.8 \%$ la calificó bien, un $24.2 \%$ la calificó como muy bien, un $5.4 \%$ la calificó regular y un $0.6 \%$ la calificó mal.

\section{Papel de los medios de comunicación}

El primer medio de comunicación por el cual la población se enteró de la epidemia fue por la radio con un $75.3 \%$, en segundo lugar está la televisión con un $50 \%$ y en tercer lugar los brigadistas de salud con $33.3 \%$. El resto de medios de comunicación presentaron porcentajes por debajo del $10 \%$.

En la actualidad la población se informa sobre la leptospirosis en primer lugar con la radio con un $67.8 \%$, seguido de la TV con un 53\%, los medios restantes presentan porcentajes por debajo del $5 \%$. El trabajo de los medios fue valorado por la población como bueno por el $74 \%$ de los encuestados y como muy bueno por el $17.3 \%$, las evaluaciones negativas estuvieron alrededor del $8.4 \%$.

De acuerdo a la población, el medio más efectivo empleado para la campaña de comunicación referente a la leptospirosis, fue la radio con un $74.3 \%$, seguido de la TV con el $56.6 \%$, los periódicos con un $3 \%$ y por personas conocidas $0.5 \%$. 


\section{Datos cualitativos (entrevista en profundidad y grupos focales)}

\section{Resultados de los grupos focales}

\section{Influenza humana: generalidades}

\begin{tabular}{|c|c|c|}
\hline $\begin{array}{l}\text { Ustedes están enterados que el } \\
\text { año pasado, en el 2010, } \\
\text { Nicaragua estuvo enfrentada a } \\
\text { un brote epidémico de } \\
\text { Leptospirosis? }\end{array}$ & $\begin{array}{l}\text { Ustedes creen la Leptospirosis en } \\
\text { Nicaragua, está controlada, fuera de } \\
\text { control, rebasó la capacidad del } \\
\text { sistema público de salud? Favor } \\
\text { expresar sus criterios. }\end{array}$ & $\begin{array}{l}\text { Ustedes creen que la } \\
\text { Leptospirosis afecta la economía } \\
\text { del país y de las familias?; favor } \\
\text { explique sus argumentos }\end{array}$ \\
\hline Si estamos enterados & $\begin{array}{l}\text { Creemos que la Leptospirosis está } \\
\text { controlada, las personas fueron } \\
\text { atendidas a la brevedad, fueron } \\
\text { atendidos por médicos, brigadistas } \\
\text { de Managua y del MINSA, se } \\
\text { preocuparon dándoles veneno para } \\
\text { eliminar las ratas e hicieron } \\
\text { campañas de limpieza. }\end{array}$ & $\begin{array}{l}\text { Nos afecta, porque al } \\
\text { enfermarnos hay más gastos y la } \\
\text { economía baja. } \\
\text { La enfermedad podría llevar a la } \\
\text { muerte y esto implica gastos y } \\
\text { nos perjudica porque también } \\
\text { tenemos otros gastos y } \\
\text { responsabilidades. } \\
\text { También nos afecta porque la } \\
\text { medicina que no hay en el centro } \\
\text { de salud tenemos que comprarla } \\
\text { personalmente. }\end{array}$ \\
\hline Si estamos enterados & $\begin{array}{l}\text { Está controlada dando el veneno } \\
\text { para las ratas y medicando a la } \\
\text { población casa a casa por } \\
\text { brigadistas de salud }\end{array}$ & $\begin{array}{l}\text { Afecta porque esta enfermedad } \\
\text { es mortal, porque si alguien se } \\
\text { muere nos perjudica en la } \\
\text { economía, ya que el } \\
\text { medicamento que no se } \\
\text { encuentra en puestos de salud } \\
\text { hay que comprarlos, por otro } \\
\text { lado las personas contestaron, } \\
\text { que también porque el gobierno } \\
\text { tiene que invertir para combatir } \\
\text { la enfermedad. }\end{array}$ \\
\hline \multicolumn{3}{|l|}{ 2. Conocimientos: } \\
\hline $\begin{array}{l}\text { ¿Cuál cree usted es la forma } \\
\text { como se adquiere la } \\
\text { Leptospirosis? }\end{array}$ & $\begin{array}{l}\text { Usted cree que si se aplica la dosis } \\
\text { contra la Leptospirosis se vuelve } \\
\text { inmune ante la enfermedad? }\end{array}$ & $\begin{array}{l}\text { Explique las medidas de } \\
\text { prevención que hay que realizar } \\
\text { para evitar la Leptospirosis }\end{array}$ \\
\hline $\begin{array}{l}\text { Se adquiere por el descuido de } \\
\text { medidas higiénicas, por medio } \\
\text { de animales domésticos dentro } \\
\text { de las casas y por no tapar los } \\
\text { alimentos La mayoría de } \\
\text { enfermos son de las } \\
\text { comunidades, porque no } \\
\text { cumplen con las medidas del } \\
\text { MINSA y porque les hace } \\
\text { falta aseo- }\end{array}$ & $\begin{array}{l}\text { No sabemos. } \\
\text { Queremos saber si hay una vacuna } \\
\text { para la Leptospirosis, ya que solo } \\
\text { conocemos las pastillas. }\end{array}$ & $\begin{array}{l}\text { No bañarse en las quebradas } \\
\text { No comer peces } \\
\text { No andar descalzos } \\
\text { Usar botas de hule } \\
\text { No tener animales } \\
\text { domésticos dentro de la casa } \\
\text { Tapar la comida } \\
\text { Asear las viviendas y patios } \\
\text { Bañarse todos los días } \\
\text { Lavar las frutas y verduras antes } \\
\text { de comerlas }\end{array}$ \\
\hline $\begin{array}{l}\text { Se contagia a través de laorina } \\
\text { de las ratas, bañándose en }\end{array}$ & $\begin{array}{l}\text { Las personas contestaron que } \\
\text { vacuna contra la leptospirosis no }\end{array}$ & $\begin{array}{l}\text { Tapando los alimentos } \\
\text { Los animales domésticos, deben }\end{array}$ \\
\hline
\end{tabular}




\begin{tabular}{|c|c|c|}
\hline $\begin{array}{l}\text { aguas contaminadas } \mathrm{de} \\
\text { animales infectados. }\end{array}$ & $\begin{array}{l}\text { hay, pero si hay pastillas para } \\
\text { medicar ala población. }\end{array}$ & $\begin{array}{l}\text { tener su lugar y no dentro de les } \\
\text { viviendas. } \\
\text { Eliminar los criaderos de ratas } \\
\text { Mantener limpias las viviendas } \\
\text { Sensibilizar a las personas para } \\
\text { que no caminen descalzos en } \\
\text { charcos, aguas estancadas, ríos, } \\
\text { quebradas. }\end{array}$ \\
\hline \multicolumn{3}{|l|}{ 3. Actitudes: } \\
\hline $\begin{array}{l}\text { Usted cree que el gobierno } \\
\text { debe asumir la total } \\
\text { responsabilidad de evitar que } \\
\text { las personas se contagien de } \\
\text { Leptospirosis? }\end{array}$ & $\begin{array}{l}\text { Actualmente usted participa de } \\
\text { actividades que permitan la } \\
\text { prevención de la Leptospirosis? Si } \\
\text { la respuesta es positiva, favor } \\
\text { explicar qué tipo de actividades y } \\
\text { cuál es la organización - institución } \\
\text { que las implementa? }\end{array}$ & $\begin{array}{l}\text { Usted se ha preocupado por } \\
\text { informarse sobre la Leptospirosis } \\
\text { y ha aplicado las medidas que se } \\
\text { aconsejan para evitar } \\
\text { enfermarse? }\end{array}$ \\
\hline $\begin{array}{l}\text { El gobierno debe asumir una } \\
\text { parte de ayuda y como } \\
\text { personas debemos tomar las } \\
\text { recomendaciones del MINSA. } \\
\text { Entonces es un compromiso } \\
\text { como persona como dice el } \\
\text { presidente Ortega "el pueblo } \\
\text { presidente" nosotros como } \\
\text { seres humanos debemos poner } \\
\text { en práctica las medidas } \\
\text { correspondientes. }\end{array}$ & $\begin{array}{l}\text { Si participamos en jornadas de } \\
\text { limpieza, distribución del veneno } \\
\text { para ratas (Biorat). } \\
\text { Las instituciones que las } \\
\text { implementa son: MINSA, Visión } \\
\text { Mundial, Alcaldía, Policía Nacional } \\
\text { y el MINED. }\end{array}$ & $\begin{array}{l}\text { Claro que sí, todos nos hemos } \\
\text { preocupado por tomarlas } \\
\text { medidas correspondientes }\end{array}$ \\
\hline $\begin{array}{l}\text { No, porque la responsabilidad } \\
\text { es de cada una de las personas, } \\
\text { aunque indicaron por otra } \\
\text { parte que el gobierno debe } \\
\text { poner de su parte para dar a } \\
\text { conocerá la población medidas } \\
\text { de prevención, y suministrar } \\
\text { medicamentos, para medicara } \\
\text { las personas contagiadas. }\end{array}$ & $\begin{array}{l}\text { Si, MINSA, Gabinete de salud del } \\
\text { poder ciudadano, MINED, Policía, } \\
\text { Juventud Sandinista, Alcaldía. }\end{array}$ & $\begin{array}{l}\text { Si porque se sensibiliza a la } \\
\text { población, para que tomaran las } \\
\text { siguientes medidas de } \\
\text { prevención: Tapar los alimentos; } \\
\text { No bañarse en charcos, ríos, } \\
\text { aguas estancadas y quebradas; no } \\
\text { dejar que los animales entrenen } \\
\text { sus viviendas; mantener limpia } \\
\text { su casa. }\end{array}$ \\
\hline \multicolumn{3}{|l|}{ 4. Practicas } \\
\hline $\begin{array}{l}\text { ¿Cuáles son las medidas que } \\
\text { usted utiliza diariamente para } \\
\text { evitar enfermarse de } \\
\text { Leptospirosis? }\end{array}$ & $\begin{array}{l}\text { ¿Se ha vacunado contrala } \\
\text { Leptospirosis? }\end{array}$ & $\begin{array}{l}\text { ¿En la casa donde usted vive, se } \\
\text { ponen en práctica medidas para } \\
\text { prevenir la Leptospirosis? Favor } \\
\text { explique }\end{array}$ \\
\hline $\begin{array}{l}\text { No dejar que los animales } \\
\text { entren a las viviendas, tapar } \\
\text { bien los alimentos, no nadar } \\
\text { en quebradas, ríos o charcos, } \\
\text { usar botas de hule. }\end{array}$ & $\begin{array}{l}\text { No existe vacuna contra la } \\
\text { Leptospirosis, solo pastillas y } \\
\text { practicar la higiene. }\end{array}$ & $\begin{array}{l}\text { Sí las ponemos en práctica. } \\
\text { Tenemos higiene, tapamos los } \\
\text { alimentos y seguimos las } \\
\text { recomendaciones del MINSA. }\end{array}$ \\
\hline $\begin{array}{l}\text { Eliminar: Charcos, basureros } \\
\text { clandestinos, limpiar los } \\
\text { predios vacíos, mantener } \\
\text { limpia su vivienda y tapar sus } \\
\text { alimentos. }\end{array}$ & $\begin{array}{l}\text { No, solo medicaron, porque vacuna } \\
\text { no existe }\end{array}$ & $\begin{array}{l}\text { Lavar frutas y verduras antes de } \\
\text { consumirlas, limpiar las } \\
\text { viviendas, taparlos alimentos, } \\
\text { evitar que los animales entrenen } \\
\text { la vivienda. }\end{array}$ \\
\hline
\end{tabular}




\begin{tabular}{|c|c|}
\hline \multicolumn{2}{|l|}{ 5. Percepción del gobierno } \\
\hline $\begin{array}{l}\text { ¿Cómo califica la reacción de las autoridades para } \\
\text { enfrentar las consecuencias de este brote } \\
\text { epidémico, ¿le parece que...? }\end{array}$ & $\begin{array}{l}\text { ¿Cómo calificaría usted el trabajo que han } \\
\text { realizado durante esta brote epidémico: La } \\
\text { presidencia de la República y el Ministerio de } \\
\text { Salud }\end{array}$ \\
\hline $\begin{array}{l}\text { Actuaron bien día a día, trabajaron, apoyaron al } \\
\text { MINSA y a las demás autoridades como la } \\
\text { Alcaldía, la Policía, Visión Mundial y el MINED } \\
\text { para que la enfermedad nose propagara, dieron una } \\
\text { información muy completa y su trabajo muy } \\
\text { eficiente. }\end{array}$ & $\begin{array}{l}\text { Su trabajo lo calificamos muy bueno, hicieron todo } \\
\text { lo que estaba en sus manos. }\end{array}$ \\
\hline $\begin{array}{l}\text { Bien porque el MINSA atendió a la brevedad, con } \\
\text { las demás instituciones correspondientes, como la } \\
\text { juventud sandinista, alcaldía, Cooperativa Juan } \\
\text { Francisco Paz Silva, MINED, Policía }\end{array}$ & $\begin{array}{l}\text { Lo califican como un trabajo eficiente. Porque la } \\
\text { presidencia de la república se preocupa por los } \\
\text { pobres del país. }\end{array}$ \\
\hline \multicolumn{2}{|l|}{ 6. Medios de comunicación. } \\
\hline $\begin{array}{l}\text { ¿Cuál es el medio que usted más utiliza para } \\
\text { informarse sobre la Leptospirosis? }\end{array}$ & $\begin{array}{l}\text { ¿Cuál es la fuente de información más creíble para } \\
\text { usted sobre la Leptospirosis? }\end{array}$ \\
\hline $\begin{array}{l}\text { Utilizamos la radio y la televisión (canal 10). } \\
\text { Noticiero 7:00 am, 1:00 pm, 7:00 pm y las } \\
\text { ediciones especiales (sábados y domingos) }\end{array}$ & Autoridades, Gobierno (MINSA). \\
\hline $\begin{array}{l}\text { Radio Ya (edición 4:00am) } \\
\text { Canal } 4 \text { (Canal Revolucionario) } \\
\text { Canal } 10 \text { (todas sus ediciones)ABC Estero (Edición } \\
\text { 6:00 am) }\end{array}$ & Autoridades del gobierno revolucionario. \\
\hline
\end{tabular}

\section{DISCUSIÓN}

\section{Comprensión de la población sobre la Leptospirosis y sus formas de transmisión}

Al observar las 10 variables utilizadas para medir la compresión de la población sobre la leptospirosis y sus formas de transmisión, predominan puntajes muy altos, lo que indica un nivel de comprensión muy alto en la mayoría de la población, un resultado relevante tomando en cuenta el alto porcentaje de la población con bajo nivel educativo (analfabeta y alfabetizada).

Analizando el comportamiento global de las variables relacionadas a este objetivo, encontramos que la variable de mayor porcentaje de comprensión, corresponde al reconocimiento de la unidad de salud como el lugar para acudir ante casos sospechosos de leptospirosis y la variable de menor porcentaje de comprensión, corresponde al reconocimiento de la leptospirosis como la razón de la alerta sanitaria.
La comprensión de una campaña de comunicación en salud depende de diversos factores, entre ellos, definir claramente el problema y señalar los objetivos; identificar la población diana; seleccionar los canales y los medios; crear los mensajes; y aplicar la evaluación en las distintas fases de la campaña (Algarra $M, 1995$ ), pero sobre todo asegurar los métodos empleados, examinar la reacción de la gente y de acuerdo a ello, rediseñar la campaña (Gómez M,1998).

Uno de los puntos básicos de la campaña, está relacionado con los mecanismos de transmisión y específicamente, "que la leptospirosis se transmite a través del contacto de las mucosas y la piel lesionada con aguas contaminadas con la orina de animales infectados con Leptospiras", solo fue identificado por un poco más de la tercera parte de los entrevistados.

Aunque las personas recuerdan que la situación de la leptospirosis fue la principal noticia en salud 
durante el período de estudio, no todos lo vinculan con el decreto de la alerta sanitaria. Debe tomarse en cuenta que los mensajes educativos escritos solo son accesibles a las dos terceras partes de la población, el perifoneo está limitado a la población que habita en el casco urbano del municipio, y no existen radios locales, aunque se escuchan radios de El Sauce y Estelí.

En síntesis, la medición "dura" muestra 5 variables con valor muy alto de comprensión de la población, 4 con un valor alto de comprensión y 1 variable con un valor bajo de comprensión, de un total de 10 variables, lo cual indica que la mayoría de la población mostró una excelente comprensión sobre la leptospirosis, aunque esto puede y debe ser mejorado con un mayor involucramiento de los agentes sociales del municipio.

La información proporcionada por los grupos focales y la entrevista en profundidad valida este resultado, con puntos de vista y valoraciones similares.

\section{Reconocimiento y prácticas de la comunidad sobre las medidas de mitigación, control y prevención recomendadas por el Ministerio de Salud}

Existe un claro predominio de prácticas de prevención muy altas en la revisión de las variables que miden esta actitud entre la población. Aunque debe destacarse que se deposita en el gobierno y MINSA toda la responsabilidad para la atención y prevención de la enfermedad, posiblemente por el enfoque biomédico que centra el abordaje de los problemas de salud en la acción de la autoridad sanitaria. También se observa una baja proporción de personas y familias que cumplen todas las medidas recomendadas por el MINSA.

La prevención de la salud, el control de la enfermedad y la atención a las personas afectadas, son los objetivos deseables de una campaña educativa, dirigida a problemas como la situación de Leptospira en Achuapa (MINSA2010, OM/OPS, 2008). Es importante destacar que mayoritariamente la población atiende las recomendaciones del MINSA y hay una actitud de contribuir en la prevención, pero no todos cumplen con las medidas orientadas. La estrategia de prevención de la salud es parte de una cultura, que aún no logra ser parte de la actividad cotidiana de la población, sobre todo porque persiste la concepción errónea que la responsabilidad es únicamente del Ministerio de Salud (Ministerio de Salud, 2008).

Asumiendo el contexto local de Achuapa, un municipio semi rural, poco accesible, una población con bajos niveles educativos y con las limitaciones propias del sistema local de salud, los resultados obtenidos son relevantes, al marcar muy alto en 5 de las 7 variables medidas en la encuesta domiciliar.

La información cualitativa también arroja resultados similares, en cuanto que la mayor parte de la comunidad pone en práctica las recomendaciones sugeridas por el personal de salud. Usualmente la tendencia en la medición de las actitudes es verificar un alto nivel de información y conocimientos, pero con prácticas de riesgo, en cambio en este estudio, hay una mayor congruencia entre los aspectos cognitivos y comportamentales, lo que indica una mayor efectividad de la estrategia de comunicación del Ministerio de Salud.

\section{Percepción que tiene la población de Achuapa referente al trabajo desempeñado por el Ministerio de Salud}

El resultado obtenido sobre el desempeño del MINSA es ambivalente, por un lado se considera que la información es correcta, oportuna y apropiada, además que asegura la atención a los casos. Por otro lado no se considera que el MINSA transmita información realista, y que su trabajo no haya sido tan exitoso como debería, más que una crítica al trabajo del MINSA, hay un llamado a un mejor desempeño, sobre todo teniendo en consideración que no se disponen de los recursos necesarios y que predomina una visión medicalizada de los problemas de salud.

La información cualitativa indica que la población reconoce la autoridad del MINSA y valora muy positivamente el trabajo realizado, aunque también 
se reconoce "que con la gente nunca se queda bien" y que más bien "depende de la actitud de las personas".

De igual manera en el grupo focal los pobladores califican de bueno y eficiente el desempeño del Ministerio de Salud. La cultura en salud en Nicaragua está influida por varias condicionantes, del contexto social y político, por lo que difícilmente se obtiene un reconocimiento unánime del trabajo desarrollado.

El Modelo de salud familiar y comunitaria de Nicaragua (MOSAFC) se enfoca precisamente en fortalecer el papel de la autoridad rectora, creando a su vez los espacios para una amplia participación comunitaria (MINSA, 2008). Los resultados obtenidos indican que una proporción importante de la población, aun espera un mayor protagonismo del MINSA, posiblemente influido por el enfoque biomédico $y$ las estrategias asistencialistas, que reducen el papel de la comunidad a simples receptores o beneficiarios de las intervenciones sanitarias.

Es responsabilidad de los agentes locales, comunitarios, sociales y religiosos del municipio, fortalecer la coordinación y la acción intersectorial e interinstitucional en busca de una acción concertada y la construcción de una agenda local de salud, donde el tema prevención y control de la leptospirosis, sea un tema permanente y transversal. Percepción que tiene la población de Achuapa
referente Papel de los medios de comunicación

El medio más utilizado habitualmente y en la actualidad ha sido la radio, lo cual es típico en las comunidades tanto rurales como en los municipios pequeños en Nicaragua, con una frecuencia de 8 de cada 10 hogares en Achuapa. La efectividad de los medios fue valorado como bajo por la mayor parte de entrevistados, una cifra que obliga a revisar la estrategia mediática de comunicación e información realizada en el municipio. Los resultados obtenidos son coherentes con el contexto del municipio, tanto en lo relativo a la accesibilidad geográfica, niveles educacionales, cultura en salud, nivel de participación comunitaria y carencia de medios de comunicación locales.

Evolución del número de casos positivos de leptospirosis, posterior a la fecha de formalización de la campaña de comunicación, en el Municipio de Achuapa

Durante el primer semestre del 2010, solo se habían presentado 4 casos confirmados de leptospirosis en el Municipio de Achuapa, sin embargo para el segundo semestre de este mismo año, se presentó un total de 43 casos, para un total de 47 casos en el 2010, número significativamente alto, al compararse con los casos presentados durante el 2008 y 2009.

Al analizar los casos acumulados de leptospirosis por semana epidemiológica, podemos apreciar que en el momento en que el gobierno declara la Alerta Sanitaria (semana 39), la curva de casos se encuentra en estado ascendente, con una alta tendencia de crecimiento de los casos acumulados de leptospirosis, sin embargo hacia la semana 42, la tendencia empieza a controlarse, aunque sigue en aumento hasta la semana 46, en donde la curva se normaliza.

De manera paralela, al analizar los casos puntuales de leptospirosis por semana epidemiológica, podemos apreciar que posterior a la declaración dela Alerta Sanitaria, y después de haberse presentado un máximo de 8 casos en la semana 39, la cual coincide con el lanzamiento de la campaña de publicidad, se aprecia un descenso moderado en las dos siguientes semanas, con una reducción significativa a partir de la semana 42.

\section{CONCLUSIONES}

Las conclusiones encontradas fueron:

1. Existe una comprensión muy alta de la población sobre la leptospirosis y sus formas de transmisión, ya que la evaluación de las variables de comprensión mostraron valores, lo cual fue ratificado por la entrevista y los resultados de los grupos focales. 
2. Existe una comprensión muy alta de la población sobre la leptospirosis y sus formas de transmisión, ya que la evaluación de las variables de comprensión mostraron valores, lo cual fue ratificado por la entrevista y los resultados de los grupos focales.

3. El reconocimiento y prácticas de la comunidad sobre las medidas de prevención y control sobre la leptospirosis, recomendadas por el Ministerio de Salud fueron valoradas muy altas, ya que se evidenció recomendaciones del MINSA. Por otra parte se evidencia un bajo cumplimiento de las medidas de prevención y control.

4. El papel de los medios de comunicación fue valorado como bajo, ya que posiblemente factores como la accesibilidad geográfica, nivel es educacionales y la carencia de los medios de comunicación, afectaron notablemente la valoración por parte de la población.

\section{BIBLIOGRAFÍA}

Algarra M. Las campañas de comunicación pública. La comunicación y salud como campo de estudio. España, 1997. En:www.unav.es/fcom/comunicacionysocied ad/.../20091106235853.pdf-Similares (revisado el 27/12/2010)

Alihuen-Argentina 2010. Leptospirosis: la dolencia de la pobreza. En:http://www.alihuen.org.ar/informacionen-general/leptospirosis.-la-dolencia-delapobreza.html (revisado el día 08/12/10)

Benenson A. Manual de Enfermedades Transmisibles en el Hombre. 16edición.OPS/OMS. Washington.

Gómez M. Teoría y práctica para la promoción de la salud. Universidad de Montreal. 1998.

Healthy People 2010. Area focus number 11: Health Communication. En:http://www.health.gov. (revisado el 25/03/2010) NTON 24 001-05, Nicaragua. Febrero del 2006. Norma
INFOMED, Vigilancia en Salud. Repositorio de artículos. Noviembre del2010. En: http://articulos.sld. cu/vigilancia/2010/11/ (revisado el 10/12/2010).

INFOMED, Vigilancia en Salud. Repositorio de artículos. Noviembre del2010. En: http://articulos.sld.cu/vigilancia/2010/11/ (revisado el 10/12/2010).

Ministerio de Salud Nicaragua. Prevengamos la Leptospirosis. Ministerio de Salud, Managua, 2010. En. http://www.minsa.gob.ni/ (revisado el27/12/2010)

Ministerio de Salud Nicaragua. Prevengamos la Leptospirosis. Ministerio de Salud, Managua, 2010. En. http://www.minsa.gob.ni/ (revisado el27/12/2010)

OPS/OMS. Leptospirosis humana: Guía para el diagnóstico, vigilancia y control. Rio de Janeiro. 2008.

OPS/OMS. Leptospirosis humana: Guía para el diagnóstico, vigilancia y control. Rio de Janeiro. 2008.

Quijano Narvaez B. Entrevista personal, diciembre 2010. Centro de Salud Achuapa. Ministerio de Salud-Nicaragua. Boletín Epidemiológico Semana 40.Managua, 2003.

Quijano Narvaez B. Entrevista personal, diciembre 2010. Centro de Salud Achuapa. Ministerio de Salud-Nicaragua. Boletín Epidemiológico Semana 40.Managua, 2003.

Salt JM: A Dictionary of epidemiology. Oxford University New York. 1988.

SINAPRED, Alerta Sanitaria. Octubre del 2010. En:http://www.sinapred.gob.ni/index.php?o ption $=$ com_content\&view $=$ article\&id $=605: e$ mergenciasanitaria\&catid=105: Boletines $\% 20$ minsaq\&Itemid=354. (revisado el 15/12/2010)nsf/\%28\$All\%29/2EB067417971 
707106257562005909CF?Open-Document (revisado el día 10/10/10).

SINAPRED, Alerta Sanitaria. Octubre del 2010. En:http://www.sinapred.gob.ni/index.php?o ption=com_content\&view=article\&id=605:e mergenciasanitaria\&catid $=105$ : Boletines $\% 20$ minsaq\&Itemid=354. (revisado el 15/12/2010)

Técnica Obligatoria de prevención y control de la leptospirosis humana. En :http://legislacion. asamblea.gob.ni/Norma web. nsf/\%28\$All\%29/2EB0674179717071062575 62005909CF?OpenDocument (revisado el día 10/10/10). 